\title{
Probability Distribution of Random Paths in the Ising Model at Low Temperature
}

\author{
Tizian R. Maren $\dagger$ \\ II. Institut für Theoretische Physik der Universität Hamburg, D-2000 Hamburg 50, \\ Federal Republic of Germany
}

\begin{abstract}
The random walk representation of the $n$-dimensional Ising model exhibits the 2-point correlation function $\langle\sigma(x) \sigma(y)\rangle$ as a sum of positive contributions of paths $\omega$ from $x$ to $y$. We derive upper bounds on the individual terms in this sum for low temperatures. Each term tends to zero as $\beta \rightarrow \infty$, while the correlation function itself tends to 1 . Therefore increasingly more and longer paths contribute when $\beta$ is lowered.
\end{abstract}

\section{Introduction}

This investigation was motivated by the Durhuus-Fröhlich [random surface] representation [1] of the Wilson loop in lattice gauge theories: The expectation value of the Wilson loop in an $n$-dimensional theory can be written as an average of the product of correlation functions of $(n-1)$-dimensional spin systems with fluctuating couplings. For each of these correlation functions one uses a random path representation of the kind that is investigated in the present paper. These paths lie in hyperplanes above each other and vary independently. They can be combined to a surface which has the Wilson loop as its boundary. The question arises what surfaces will make a nonnegligible contribution to the Wilson loop at a given temperature [coupling constant]. For instance one will expect that the confining (high temperature) phase and the Higgs (low temperature) phase of a pure $\mathbb{Z}_{2}$ lattice gauge theory in 3 or 4 dimensions [2] will be characterized by a qualitatively different probability distribution of these random surfaces (compare [3]).

In this paper the corresponding question for the correlation function of the Ising model is investigated. A qualitatively different behavior at high and low temperatures is found. At high temperatures it is immediately seen from the random path representation that the shortest path makes the dominant contribution. The main problem was therefore to analyse the multiple integrals that appear in the random path representation at low temperatures. This problem was solved with the help of a combination of high temperature (Mayer-) and low 
temperature (Peierls-) expansions, following the method of Bricmont et al. [4]. It turns out that the contribution of each individual path vanishes in the limit of low temperatures $(T \rightarrow 0)$. Therefore more and more, and consequently increasingly longer, paths must contribute to the correlation function when the temperature is lowered.

\section{Random Walk Representation of the Correlation Function in the Ising Model}

We consider an Ising ferromagnet on a finite hypercubic lattice $\Lambda \subset \mathbb{Z}^{n}$ with free boundary conditions in $n$ dimensions. Here $\Lambda$ is chosen as a cube of side length $\lambda$. A configuration $\sigma \in \mathbb{Z}_{2}^{\Lambda}$ fixes the values $\sigma(x)=+1$ (spin up) or -1 (spin down) of the spin variables attached to every site $x$ of the lattice $\Lambda$. The set $\Lambda_{l}$ of links $l$ in $\Lambda$ consists of pairs $l=\{x, y\}$ of nearest neighbor sites in $\Lambda$. We define $\sigma(l)=\sigma(x) \sigma(y)$ for such a link. The expectation value $\langle A\rangle^{\beta, A}$ of the observable $A$ at inverse temperature $\beta$ on the lattice $\Lambda$ is defined by

$$
\begin{aligned}
\langle A\rangle^{\beta, \Lambda} & =\frac{1}{Z} 2^{-|\Lambda|} \sum_{\sigma} A(\sigma) e^{-\beta E(\sigma)}, \\
Z \equiv Z^{\beta, \Lambda} & =2^{-|A|} \sum_{\sigma} e^{-\beta E(\sigma)} ; \quad E(\sigma)=-\sum_{l \in \Lambda_{l}} \sigma(l) .
\end{aligned}
$$

A path $\omega$ of length $n=|\omega|$ is a sequence of $n$ sites $\omega_{1}, \ldots, \omega_{n}$ such that $\left\{\omega_{i}, \omega_{i+1}\right\}$ is a pair of nearest neighbours on $\Lambda$. We denote by $\Omega_{x y}$ the set of all such paths of arbitrary length between $x=\omega_{1}$ and $y=\omega_{n}$. Here $n_{\omega}(z)$ is the number of visits of the path $\omega$ to the site $z$, and $\bar{\omega}$ is the set of distinct sites that are visited by $\omega$. The length $|\omega|$ of the path and the number $\|\omega\|$ of distinct points on the path $\omega$ are

$$
|\omega|=\sum_{z \in \bar{\omega}} n_{\omega}(z) ; \quad\|\omega\|=\sum_{z \in \bar{\omega}} 1
$$

The random walk representation of the 2-point correlation function reads $[1]^{1}$

$$
\begin{aligned}
Z\left\langle\sigma\left(x_{0}\right) \sigma\left(y_{0}\right)\right\rangle= & \sum_{\omega}\left(\frac{1}{2}\right)^{|\omega|} \beta^{|\omega|-1} \prod_{z \in \bar{\omega}}\left[\left(n_{\omega}(z)-1\right) !\right]^{-1} \\
& \cdot \int_{\mathbb{Z}_{2}} \prod_{y \in \Lambda \backslash \bar{\omega}} d s_{y} \int_{-1}^{+1} \prod_{z \in \bar{\omega}}\left(1-s_{z}^{2}\right)^{n_{\omega}(z)-1} d s_{z} \exp \left[\beta \sum_{l \in \Lambda_{l}} s(l)\right] .
\end{aligned}
$$

Summation runs over all paths $\omega \in \Omega_{x_{0} y_{0}}$ from $x_{0}$ to $y_{0}$. Variables $s_{y}$ attached to sites $y$ on the path $\omega$ are integrated over the real interval $0 \ldots 1$, while for sites $y$ not on the path they are averaged over the two values +1 and -1 .

The factor $\beta^{|\omega|-1}$ in Eq. (2.3) will prove to be essential in the high- and low temperature expansion.

By rescaling the spins $s_{y}$ with $y \in \bar{\omega}$ with the help of new variables $\tilde{\beta}_{x} \in[0,1]$ one can regain an Ising model. The contribution of a path $\omega$ appears then as an

1 A comprehensive introduction to random walk representations of classical spin systems was recently given by Brydges et al. Related to these ideas is the work of Aizenman. For references, see [5] 
average over Ising models with fluctuating coupling strengths $\beta \tilde{\beta}(l)$; the average is weighted by Poisson distributions. $\tilde{\beta}(l) \equiv \tilde{\beta}_{x} \tilde{\beta}_{y}$ for $l=\{x, y\}$.

The variables $\tilde{\beta}_{x}$ with $x \in \bar{\omega}$ will be integrated from $0 \ldots 1$, while we set $\tilde{\beta}_{x}=1$ for sites $x$ which are not on the path $\omega$. Equation (2.3) takes the form

$$
\left\langle\sigma\left(x_{0}\right) \sigma\left(y_{0}\right)\right\rangle^{\beta, \Lambda}=\sum_{\omega} \varrho_{\beta}(\omega) \frac{Z_{\omega}}{Z} \equiv \sum_{\omega} \mathscr{P}\left(n_{\omega}\right),
$$

with

$$
\begin{aligned}
\varrho_{\beta}(\omega) & =\beta^{|\omega|-1}\left(\frac{1}{2}\right)^{|\omega|-\|\omega\|} \prod_{z \in \bar{\omega}}\left(\left[n_{\omega}(z)-1\right] !\right)^{-1}, \\
Z_{\omega} & =\int_{0}^{1} \prod_{z \in \bar{\omega}} d \tilde{\beta}_{z}\left(1-\tilde{\beta}_{z}^{2}\right)^{n_{\omega}(z)-1}\left(\frac{1}{2}\right)^{|\Lambda|} \sum_{\sigma \in \mathbb{Z}_{2}^{\Lambda}} \exp \left[\beta \sum_{l \in \Lambda_{l}} \tilde{\beta}(l) \sigma(l)\right] .
\end{aligned}
$$

The contribution of a path $\omega$ depends on $\omega$ through the numbers of visits $n_{\omega}(x)$ to the sites $x$ of $\Lambda$. Therefore we denote it by $\mathscr{P}\left(n_{\omega}\right)$.

It is straightforward to extend the representation (2.4) to the case of + boundary conditions (spins up outside $\Lambda$ ). For $x \in \Lambda$ let $h_{x}$ denote the numbers of nearest neighbours of $x$ in $\mathbb{Z}^{n}$ which do not lie in $\Lambda$. Then the result for + boundary conditions reads

$$
\langle\sigma(x) \sigma(y)\rangle^{\beta, \Lambda}=\sum_{\substack{\omega \\ \omega \in \Omega_{x y}}} \mathscr{P}^{+}\left(n_{\omega}\right)+\beta \sum_{z \in \Lambda} \sum_{z^{\prime} \in \Lambda} \sum_{\substack{\omega_{1} \\ \omega_{1} \in \Omega_{x z}}} \sum_{\substack{\omega_{2} \\ \omega_{2} \in \Omega_{z^{\prime} y}}} h_{z} h_{z^{\prime}} \mathscr{P}\left(n_{\omega_{1}}+n_{\omega_{2}}\right) .
$$

Here $\mathscr{P}^{+}$is given by the same expression as $\mathscr{P}$, except that partition functions are to be computed with + boundary conditions.

It is instructive to consider the simplest example of a lattice $\Lambda$ of only two (neighbouring) points $x$ and $y$, with free boundary conditions. The contribution of the (only) path of length $2 k+2$ is found to be

$$
\mathscr{P}=\frac{1}{\cosh \beta} \beta^{2 k+1} \sum_{n=0}^{\infty} \beta^{2 n} \frac{1}{(2 n) !}\left(\frac{(2 n-1) ! !}{(2 n+2 k+1) ! !}\right)^{2} .
$$

When this is plotted one sees very distinctly that only the shortest path $(k=0)$ makes an essential contribution for high temperatures (small $\beta$ ), while for $\beta \rightarrow \infty$ the contribution of any individual path tends to zero, so that increasingly more and longer paths contribute to the correlation function $\langle\sigma(x) \sigma(y)\rangle^{\beta, \Lambda}=\tanh \beta$ for large $\beta$.

\section{Expansions}

We will interpret $Z$ and $Z_{\omega}$ as partition functions of suitably defined polymer systems (in the sense of Gruber and Kunz [6]). We proceed in two steps. First we replace $\sum_{\sigma}$ by a Peierls expansion. A configuration $\sigma$ determines a set $P$ of links $l$ where $\sigma(l)=-1$. Here $P$ is coclosed, i.e. every plaquette on $\Lambda$ contains an even number of links in $P$. Such $P$ are called Peierls contours. Conversely, every Peierls 
contour determines a configuration $\sigma$ uniquely up to a global $\mathbb{Z}_{2}$-transformation $\sigma(x) \rightarrow-\sigma(x)$. The Peierls expansion reads [7]

$$
\begin{aligned}
Z & =2 e^{\beta\left|\Lambda_{l}\right|}\left(\frac{1}{2}\right)^{|\Lambda|} \sum_{P} e^{-2 \beta|P|} \equiv 2 e^{\beta\left|\Lambda_{l}\right|}\left(\frac{1}{2}\right)^{|\Lambda|}\left(Z_{I}\right)_{\text {Poly }}, \\
Z_{\omega} & =2 e^{\beta\left|\Lambda_{l}\right|}\left(\frac{1}{2}\right)^{|\Lambda|} \sum_{P} \int_{0}^{1} \prod_{z \in \bar{\omega}} d \tilde{\beta}_{z}\left(1-\tilde{\beta}_{z}^{2}\right)^{n_{\omega}(z)-1} \prod_{l \in P} e^{-\beta(\tilde{\beta}(l)+1)} \prod_{l \notin P} e^{\beta(\tilde{\beta}(l)-1)} .
\end{aligned}
$$

Summation is over all Peierls contours $P$ on $\Lambda$.

The second step is a Mayer expansion for the $\tilde{\beta}$-integral in $Z_{\omega}$. We set

$$
\tilde{\beta}_{x}^{\prime}=1-\tilde{\beta}_{x}
$$

and consider $\tilde{\beta}_{x}^{\prime}$ as the variable of integration if $x \in \Lambda \backslash \hat{P}$. Here $\hat{P}$ is the set of sites on the path $\omega$ which belong to a link in $P$. [This substitution is motivated by the fact that if $l=\{x, y\}$ is not in $P$, then $\sigma(l)=+1$ and $e^{\beta \tilde{\beta}(l) \sigma(l)}$ is maximal for $\tilde{\beta}_{x}=\tilde{\beta}_{y}=1$.] For a point $z$ on the path $\omega$ we denote by $c_{z}$ the number of links (in $\Lambda_{l}$ ) which contain $z$ and do not belong to $P$. Using the substitution (3.2), we obtain from (3.1b)

$$
\begin{aligned}
Z_{\omega}= & 2\left(\frac{1}{2}\right)^{|\Lambda|} \exp \left[\beta\left|\Lambda_{l}\right|\right] \sum_{P} \int_{0}^{1} \prod_{z \in \hat{P}} d \tilde{\beta}_{z}\left(1-\tilde{\beta}_{z}^{2}\right)^{n_{\omega}(z)-1} \\
& \cdot \int_{0}^{1} \prod_{y \in \bar{\omega} \backslash \hat{P}} d \tilde{\beta}_{y}^{\prime}\left(2 \tilde{\beta}_{y}^{\prime}-\tilde{\beta}_{y}^{\prime 2}\right)^{n_{\omega}(y)-1} \prod_{l \in P} e^{-\beta(\tilde{\beta}(l)+1)} \\
& \cdot \prod_{l \notin P}\left(e^{\beta \tilde{\beta}^{\prime}(l)}-1+1\right) \prod_{z \in \bar{\omega}} \exp \left[-\beta c_{z} \tilde{\beta}_{z}^{\prime}\right] .
\end{aligned}
$$

The reader is asked to bear in mind that $\tilde{\beta}_{x}^{\prime}$ stands for $1-\tilde{\beta}_{x}$ for points $x$ which belong to $\hat{P}$. Therefore the above integral does not factorize into $\int \prod_{z}(\ldots)$ and $\int \prod_{y}(\ldots)$. Convergence of the Mayer expansion below will come from the last factor in (3.3).

Now we may apply the Mayer expansion

$$
\prod_{x \in \mathscr{M}}\left(1+\mathscr{A}_{x}\right)=\sum_{X \subset \mathscr{M}} \prod_{x \in X} \mathscr{A}_{x} .
$$

Since $\tilde{\beta}_{x}^{\prime}=0$ for sites $x$ which do not belong to the path $\omega$, we may restrict summation to sets $X$ of links that lie on the path $\omega$.

$$
X \subset \bar{\omega}_{l} \equiv\left\{l \in \Lambda_{l}, l \subset \bar{\omega}\right\}
$$

The product to be expanded runs only over links $l \notin P$. Therefore in the Mayer expansion we need only sum over subsets $X$ of $\bar{\omega}_{l} \backslash P$. This gives

$$
Z_{\omega}=2\left(\frac{1}{2}\right)^{|\Lambda|} e^{\beta\left|\Lambda_{l}\right|} F(\bar{\omega})\left(Z_{\omega}\right)_{\text {Poly }}
$$


with

$$
\begin{aligned}
\left(Z_{\omega}\right)_{\mathrm{Poly}}= & 1+\sum_{P, X} A_{\omega}(P, X), \\
F(A)= & \prod_{x \in A} \int_{0}^{1} d \tilde{\beta}_{x}^{\prime}\left(2 \tilde{\beta}_{x}^{\prime}-\tilde{\beta}_{x}^{\prime 2}\right)^{n_{\omega}(x)-1} e^{-2 n \beta \tilde{\beta}_{x}^{\prime}} \text { for } A \subseteq \bar{\omega}, \\
A_{\omega}(P, X)= & F(\hat{P} \cup \hat{X})^{-1} \int_{0}^{1} \prod_{z \in \hat{P}} d \tilde{\beta}_{z}\left(1-\tilde{\beta}_{z}^{2}\right)^{n_{\omega}(z)-1} \\
& \cdot \int_{0}^{1} \prod_{y \in \hat{X} \backslash \hat{P}} d \tilde{\beta}_{y}^{\prime}\left(2 \tilde{\beta}_{y}^{\prime}-\tilde{\beta}_{y}^{\prime 2}\right)^{n_{\omega}(y)-1} \prod_{l \in P} e^{-\beta(\tilde{\beta}(l)+1)} \\
& \cdot \prod_{l \in X}\left(e^{\beta \tilde{\beta}^{\prime}(l)}-1\right) \prod_{x \in \hat{X} \cup \hat{P}} e^{-\beta c_{x} \tilde{\beta}_{x}^{\prime}} .
\end{aligned}
$$

In the sum over $P, X$ in $(3.4 \mathrm{~b})$, the term with $P=X=\emptyset$ is to be omitted (it contributes the extra 1 ). Thus $\hat{X}$ is the set of sites on $\omega$ which belong to at least one link in $X$.

Now we are ready to interpret the partition functions $\left(Z_{I}\right)_{\mathrm{Poly}}(\propto Z)$, and $\left(Z_{\omega}\right)_{\text {Poly }}\left(\propto Z_{\omega}\right)$ as partition functions of polymer systems. Let us first consider the Peierls expansion (3.1a) of $\left(Z_{I}\right)_{\text {Poly }}$. Two Peierls contours (coclosed sets of links) $P_{1}$ and $P_{2}$ are called disjoint if there exists no plaquette (elementary square) which contains link(s) both in $P_{1}$ and in $P_{2}$. Here $P$ is called connected if it cannot be split into two disjoint Peierls contours $P_{1}$ and $P_{2}$. Temporarily, connected Peierls contours will be called polymers. Every Peierls contour $P$ can be decomposed uniquely into connected pieces (polymers) $\mathbf{P}_{1}, \ldots, \mathbf{P}_{n}$. The factor $e^{-2 \beta|P|}$ splits into a product of activities

$$
A_{I}\left(\mathbf{P}_{i}\right)=e^{-2 \beta\left|\mathbf{P}_{\imath}\right|},
$$

and the Peierls expansion (3.1a) becomes

$$
\left(Z_{I}\right)_{\mathrm{Poly}}=1+\sum_{\mathbb{P}} \prod_{\mathbf{P} \in \mathbb{P}} A_{I}(\mathbf{P}) .
$$

Summation is over nonempty collections $\mathbb{P}$ of mutually disjoint polymers (= connected Peierls contours) $\mathbf{P}$.

Next we proceed in the same way for $\left(Z_{\omega}\right)_{\text {Poly }}$. Consider pairs $(P, X)$, where $P$ is a Peierls contour and $X$ is a set of links that lie on the path $\omega$. We call $(P, X)$ and $\left(P^{\prime}, X^{\prime}\right)$ disjoint if they have no site in common (that is, if $\left.(\hat{P} \cup \hat{X}) \cap\left(\hat{P}^{\prime} \cup \hat{X}^{\prime}\right)=\emptyset\right)$. Now, $(P, X)$ is called connected, or a polymer, if it is nontrivial (i.e. $P$ or $X$ or both are nonempty), and $(P, X)$ cannot be decomposed into two disjoint pieces $\left(P_{1}, X_{1}\right)$ and $\left(P_{2}, X_{2}\right)$. From expression (3.4a) we see that $A_{\omega}(P, X)$ factors on disjoint arguments. Therefore Eq. (3.4b) gives

$$
\left(Z_{\omega}\right)_{\mathrm{Poly}}=1+\sum_{\mathbb{P}} \prod_{\mathbf{P} \in \mathbb{P}} A_{\omega}(\mathbf{P})
$$

with activities $A_{\omega}(P, X)$ as defined by Eq. (3.4d). Summation is over nonempty collections $\mathbb{P}$ of disjoint polymers $\mathbf{P}=(P, X)$. Equations (3.5) and (3.6) are the desired representations as partition functions of polymer systems in the sense of Gruber and Kunz (with monomer activity 1). 


\section{Estimates of Activities $A_{\omega}$ at Low Temperatures}

We will show that the activities $A_{\omega}$ decrease exponentially with the size of the polymer. Define $\beta_{c}(\omega)>1$ by the equation $\beta_{c}(\omega)=(1+|\omega|) \ln \beta_{c}(\omega)$. It is claimed that there exists a constant $K_{\omega}$ such that for all $\beta>\beta_{c}(\omega)$

$$
A_{\omega}(P, X) \leqq\left\{\begin{array}{lll}
K_{\omega} 5^{|\hat{X} \cup \hat{P}|} e^{-\beta(|P|-1)} \beta^{-|X|} & \text { if } \quad P \neq \emptyset \text { (i.e. }|P| \geqq 2 n), \\
K_{\omega} 5^{|\hat{X}|} \beta^{-|X|} & \text { if } \quad P=\emptyset .
\end{array}\right.
$$

First we bound $|F(\hat{X} \cup \hat{P})|$ from below. From its definition

$$
\begin{aligned}
|F(\hat{X} \cup \hat{P})| & \geqq \prod_{x \in \hat{X} \cup \hat{P}} \int_{0}^{1} d \tilde{\beta}_{x} \tilde{\beta}_{x}^{n_{\omega}(x)-1} e^{-2 n \beta \tilde{\beta}_{x}} \\
& \geqq \prod_{x \in \hat{X} \cup \hat{P}} \frac{1}{5}(2 n \beta)^{-n_{\omega}(x)} \quad \text { if } \quad \beta>1 .
\end{aligned}
$$

Here $F(\hat{X} \cup \hat{P})^{-1}$ increases with a $\omega$-dependent power of $\beta$. We will show that this will be more than compensated by the other factor in $A_{\omega}$. Let

$$
\begin{aligned}
H= & \int_{0}^{1} \prod_{z \in \hat{P}} d \tilde{\beta}_{z}\left(1-\tilde{\beta}_{z}^{2}\right)^{n_{\omega}(z)-1} \int_{0}^{1} \prod_{y \in \hat{X} \backslash \hat{P}} d \tilde{\beta}_{y}^{\prime}\left(2 \tilde{\beta}_{y}^{\prime}-\tilde{\beta}_{y}^{\prime 2}\right)^{n_{\omega}(y)-1} \\
& \cdot \prod_{l \in P} e^{-\beta(\tilde{\beta}(l)+1)} \prod_{l \in X}\left(e^{\beta \tilde{\beta}^{\prime}(l)}-1\right) \prod_{x \in \hat{X} \cup \hat{P}} e^{-\beta c_{x} \tilde{\beta}_{x}^{\prime}}
\end{aligned}
$$

The factor $\prod \mathrm{e}^{-\beta(\tilde{\beta}(l)+1)}$ depends on the Peierls contour. It will be bounded by $e^{-\beta|P|}$. Moreover, $1-\tilde{\beta}_{x}^{2}=2 \tilde{\beta}_{x}^{\prime}-\tilde{\beta}_{x}^{\prime 2} \leqq 2 \tilde{\beta}_{x}^{\prime}$. Therefore

$$
H \leqq e^{-\beta|P|} \int_{0}^{1} \prod_{x \in \hat{X} \cup \hat{P}} d \tilde{\beta}_{x}^{\prime}\left(2 \tilde{\beta}_{x}^{\prime}\right)^{n_{\omega}(x)-1} \prod_{l \in X}\left(e^{\beta \tilde{\beta}^{\prime}(l)}-1\right) \prod_{x \in \hat{X} \cup \hat{P}} e^{-\beta c_{x} \tilde{\beta}_{x}^{\prime}} .
$$

One has $e^{x}-1 \leqq x e^{x}$ for $x>0$. Let $d_{z}$ be the number of links in $X$ which contain $z$. Then $d_{z} \leqq c_{z}$. Therefore

$$
\begin{aligned}
& H \leqq e^{-\beta|P|} \int_{0}^{1} \prod_{x \in \tilde{X} \cup \hat{P}} d \tilde{\beta}_{x}^{\prime} 2^{\sum^{x}\left(n_{\omega}(x)-1\right)} \beta^{|X|} \prod_{\xi \in \hat{X}} \tilde{\beta}_{\xi}^{\prime\left(d_{\xi}+n_{\omega}(\xi)-1\right)} e^{-\beta c_{\xi} \tilde{P}_{\xi}^{\prime}} \\
& \cdot \prod_{l \in X} e^{\beta \tilde{\beta}^{\prime}(l)} \prod_{z^{\prime} \in \hat{E} \backslash \hat{X}} e^{-\beta c_{z^{\prime}} \tilde{\beta}_{z^{\prime}}\left(\tilde{\beta}_{z^{\prime}}^{\prime}\right)^{n_{\omega}\left(z^{\prime}\right)-1} .}
\end{aligned}
$$

The factor involving sites $z^{\prime}$ in $\hat{P} \backslash \hat{X}$ can only be bounded by 1 because $c_{z^{\prime}}$ may be zero. (This happens when $z^{\prime}$ is entirely surrounded by links of the Peierls contour.) Since $0 \leqq \tilde{\beta}_{z} \leqq 1$ one has $\tilde{\beta}_{x} \tilde{\beta}_{y} \leqq \frac{1}{2}\left(\tilde{\beta}_{x}+\tilde{\beta}_{y}\right)$. We insert this, write $\tilde{\beta}^{\prime}$ for $\beta \tilde{\beta}^{\prime}$ and use that $c_{\xi} \geqq 1$ if $\xi \in \hat{X}$. Thus

$$
H \leqq e^{-\beta|P|} 2^{\sum^{\left(n_{\omega}(x)-1\right)}} \beta^{-|X|} \prod_{\xi \in \hat{X}} \beta^{-n_{\omega}(\xi)} \int_{0}^{\beta} d \tilde{\beta}_{\xi}^{\prime} \tilde{\beta}_{\xi}^{\prime d d_{\xi}+n_{\omega}(\xi)-1} e^{-\frac{1}{2} c \xi \beta \xi \xi} .
$$

One has $\sum_{x \in \hat{X}} d_{x}=2|X|$. In $n$ space dimensions we define

$$
K_{\omega}=(2 n)^{|\omega|} 2^{2(|\omega|+\|\omega\|} \prod_{z \in \bar{\omega}}\left(2 n+n_{\omega}(z)-1\right) !
$$


Then the inequalities for $F(\hat{X} \cup \hat{P})$ and $H$ combine to

$$
A_{\omega}(P, X) \leqq 5^{|\hat{X} \cup \hat{P}|} K_{\omega} e^{-\beta|P|} \beta^{-|X|} \beta \xi \sum_{\xi \in \hat{P}}^{n_{\omega}(x)} .
$$

This proves (4.1) for $P=\emptyset$. If $P \neq \emptyset$ we estimate the last factor by $\beta^{|\omega|}$. If $\beta>\beta_{c}>1$ and $\beta_{c}=(1+|\omega|) \ln \beta_{c}$, then $\beta^{-|\omega|} e^{-\beta|P|} \leqq e^{-\beta[|P|-1]}$. This proves inequality (4.1).

\section{Thermodynamic Limit}

We combine the result (3.4) with the Peierls expansion (3.1a) for the partition function $Z$ of the Ising model, viz.

$$
Z=2\left(\frac{1}{2}\right)^{|A|} e^{\beta\left|A_{l}\right|}\left(1+\sum_{P \neq \emptyset} e^{-2 \beta|P|}\right) \equiv 2\left(\frac{1}{2}\right)^{|A|} e^{\beta\left|\Lambda_{l}\right|}\left(Z_{I}\right)_{\mathrm{Poly}} .
$$

From the definition (3.4c) of $F(\omega)$ one obtains easily the bound

$$
F(\bar{\omega}) \leqq 2^{|\omega|-\|\omega\|}(2 n \beta)^{-|\omega|} \prod_{z \in \bar{\omega}}\left(n_{\omega}(z)-1\right) ! .
$$

Therefore the contribution $\mathscr{P}\left(n_{\omega}\right)$ of a path $\omega$ to the 2-point correlation function (2.4) is boundary by

$$
\mathscr{P}\left(n_{\omega}\right)=\varrho_{\beta}(\omega) F(\bar{\omega}) \frac{\left(Z_{\omega}\right)_{\text {Poly }}}{\left(Z_{I}\right)_{\text {Poly }}} \leqq(2 n)^{-|\omega|} \frac{\left(Z_{\omega}\right)_{\text {Poly }}}{\left(Z_{I}\right)_{\text {Poly }}} \frac{1}{\beta} .
$$

The two partition functions have been exhibited as partition functions of polymer systems in Sect. 3. Their activities are bounded by Eqs. (3.5a) and (4.1). The free energy of a polymer system admits a linked cluster expansion [6]

$$
\ln Z=\sum_{Q} a(Q) A(Q)
$$

with

$$
A\left(\mathbf{P}_{1}^{n_{1}}, \ldots, \mathbf{P}_{k}^{n_{k}}\right)=\prod_{j} A\left(\mathbf{P}_{j}\right)^{n_{J}}
$$

Summation is over all clusters $Q=$ sets of not necessarily distinct polymers which are linked in the sense that they cannot be decomposed into two clusters made of disjoint polymers. Here $a(Q)$ are combinatorial coefficients which are independent of the lattice and the activities. Standard estimates [6] give a bound on the sum in (5.2), given estimates on the activities. It follows from our estimates (3.5a) and (4.1) that

$$
\frac{\left(Z_{\omega}\right)_{\text {Poly }}}{\left(Z_{I}\right)_{\text {Poly }}} \rightarrow 1 \text { as } \beta \rightarrow \infty
$$


uniformly in the size of the lattice $\Lambda$, but nonuniformly in $\omega$. Bound (5.1) together with (5.3) is our final result. It shows that the contribution of every single path $\omega$ tends to zero as $\beta \rightarrow \infty$.

\section{References}

1. Durhuus, B., Fröhlich, J.: A connection between $v$-dimensional Yang-Mills theory and $(v-1)$ dimensional, non-linear $\sigma$-models. Commun. Math. Phys. 75, 103 (1980)

2. Wegner, F.: Duality in generalized Ising models and phase transitions without local order parameters. J. Math. Phys. 12, 2259 (1971)

3. Wilson, K.: Cargèse 1976

4. Bricmont, J., Lebowitz, J.L., Pfister, C.E.: Low temperature expansion for continuous-spin Ising models. Commun. Math. Phys. 78, 117 (1980)

5. Brydges, D., Fröhlich, J., Spencer, T.: The random walk representation of classical spin systems and correlation inequalities. Commun. Math. Phys. 83, 125-152 (1982)

Aizenman, M. : Proof of the triviality of $\phi_{d}^{4}$ field theory and some mean field features of Ising models for $d>4$. Phys. Rev. Lett. 47, 1 (1981), and Geometric analysis of $\phi^{4}$ fields and Ising models (Parts I and II). Commun. Math. Phys. 86, 1-48 (1982)

6. Gruber, C., Kunz, H.: General properties of polymer systems. Commun. Math. Phys. 22, 133 (1971) Martin-Löf, A.: On the spontaneous magnetization in the Ising model. Commun. Math. Phys. 24, 253 (1972)

7. Peierls, R.: On Ising's model of ferromagnetism. Proc. Comb. Philos. Soc. 32, 477 (1936)

Ruelle, D.: Statistical mechanics : rigorous results. New York: Benjamin 1969

Communicated by G. Mack

Received August 29, 1982 